$24.06 \pm 8.9 \mathrm{~kg}, \mathrm{p}=0.005)$ and RLF $13.44 \pm 7.43$ vs $24.26 \pm 7.65 \mathrm{~kg}, \mathrm{p}=0.0003$ ). Patients with VFs lose their balance faster during one-leg-standing test with open eyes $(5.0[1.0 ; 10.0]$ vs $7.5[5.0 ; 10.5] \mathrm{sec}$ in control group, $p=0.03)$ and with closed eyes $(2.0[0 ; 3.0]$ vs $3.5[3.0 ; 5.0] \mathrm{sec}, \mathrm{p}=0.04)$. Fukuda-Unterberger test showed greater side dislocation in study group $-40^{\circ}[25 ; 45]$ vs controls $30^{\circ}[10$; $45],(p=0.02)$. According to stabilometry study group was characterized vs control group by lower balance coefficient with open eyes $(77.2 \pm 7.6$ vs $85.7 \pm 9.4 \%$, $\mathrm{p}=0.002)$ and with closed eyes $(67.1 \pm 9.8$ vs $73.4 \pm 9.9 \%, p=0.03)$, greater sagittal displacement $(6.8[2.1 ; 37.7]$ vs $4.8[1.8 ; 10.7] \mathrm{mm}, \mathrm{p}=0.025)$ and deviation in the saggital plane $(1.2[-1.07 ; 1.5]$ vs $-1.2[-1.5 ; 1.2] \mathrm{mm}, \mathrm{p}=0.01)$, and also less pressure center velocity $(9.51 \pm 4.4$ vs $7.1 \pm 2.7 \mathrm{~mm} / \mathrm{sec}, \mathrm{p}=0.009)$.

Conclusion: Osteoporotic VFs are associated with reduction of trunk muscles strength and negatively affect static and dynamic balance function that should be taken into account when developing rehabilitation programs for these patients.

Disclosure of Interests: None declared.

DOI: 10.1136/annrheumdis-2021-eular.4260

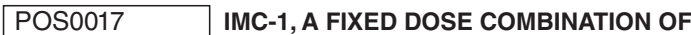 FAMCICLOVIR AND CELECOXIB, IMPROVES COMMON SYMPTOMS ASSOCIATED WITH FIBROMYALGIA IN ADDITION TO PAIN: POST HOC ANALYSIS OF A PHASE 2A TRIAL}

W. Pridgen ${ }^{1}$, C. Duffy ${ }^{2}$, J. F. Gendreau ${ }^{3}$, R. M. Gendreau ${ }^{4}{ }^{1}$ Tuscaloosa Surgical Associates, P.C., LLC, Tuscaloosa, United States of America; ${ }^{2}$ University of Alabama, Department of Biological Sciences, Tuscaloosa, United States of America; ${ }^{3}$ Gendreau Consulting, LLC, Poway, United States of America; ${ }^{4}$ Virios Therapeutics, Alpharetta, United States of America

Background: Fibromyalgia is a chronic disease characterized by widespread pain and severe fatigue that may be triggered by reactivation of latent herpes simplex virus type 1 (HSV-1). In a Phase 2a proof of concept trial, IMC-1 (a fixed dose combination of famciclovir and celecoxib) demonstrated greater tolerability and statistically significant reduction in pain compared with placebo, as measured by change from baseline to week 16 in 24-hour recall pain intensity on an 11-point Numerical Rating Scale (NRS) and 7-day recall pain intensity on the 11-point pain item on the Revised Fibromyalgia Impact Questionnaire (FIQ-R). Objectives: In this post hoc analysis, we evaluated the effects of IMC-1 compared with placebo on other fibromyalgia symptoms, including lack of energy, stiffness, problems with sleep, problems with memory, depression, and anxiety. Methods: In the double-blind, multi-center, placebo-controlled trial, male or female patients 18-70 years of age who met diagnostic criteria for fibromyalgia and had at baseline a 24-hour recall average pain intensity score between 4 and 9 on the NRS were randomized 1:1 to 16 weeks of treatment with IMC-1 or placebo. Mean changes from baseline to week 16 in FIQ-R symptom scores were analyzed using a Mixed-Effect Model Repeated Measure (MMRM) model with treatment as the main effect, and investigative site and baseline FIQ-R symptom scores as covariates. Results: A total of 143 patients were enrolled and randomized to treatment with IMC-1 $(n=69)$ or placebo $(n=74)$. Baseline demographic and clinical characteristics were comparable between treatment groups; the majority of patients were Caucasian $(95.8 \%)$ and female $(93.7 \%)$ with a mean age of $\sim 49$ years. Compared with placebo, treatment with IMC-1 resulted in statistically significant improvements in the FIQ-R symptom scores of stiffness (least squares mean change from baseline -0.96 vs. $-1.92, P=0.03)$, sleep quality $(-0.76$ vs. $-1.76, P=0.039)$, depression $(-0.44$ vs. $-1.33, P=0.016)$, and anxiety $(-0.30$ vs. $-1.69, P<0.001)$, but not in energy level (-0.67 vs. $-1.29, P=0.115)$ or memory problems $(-0.71$ vs. $-1.24, P=0.165)$.

Conclusion: In addition to alleviation of chronic pain, treatment with IMC-1 appears to be effective in improving many of the other symptoms often associated with fibromyalgia. Further clinical trials are warranted.

REFERENCES:

[1] Pridgen WL, Duffy C, Gendreau JF, Gendreau RM. A famciclovir + celecoxib combination treatment is safe and efficacious in the treatment of fibromyalgia. J Pain Res. 2017;10:451-460.

Disclosure of Interests: William Pridgen Consultant of: Virios Therapeutics, Carol Duffy Consultant of: Virios Therapeutics, Grant/research support from: The University of Alabama, Department of Biological Sciences has received financial research support from Innovative Med Concepts (now Virios Therapeutics) in the form of two Sponsored Research Agreements., Judy F. Gendreau Consultant of: Tonix, Dare Bioscience, Virios Therapeutics, R. Michael Gendreau Consultant of: Tonix, Teva, Swing Therapeutics, Dare Bioscience, Employee of: Virios Therapeutics.

DOI: 10.1136/annrheumdis-2021-eular.1424

\section{POS0018 INVESTIGATING VIRTUAL IMMERSIVE EXPERIENCES IN THE MANAGEMENT OF CHRONIC PAIN - THE VIPA STUDY (PRELIMINARY RESULTS)}

J. Tsigarides ${ }^{1}$, V. Grove ${ }^{1}$, D. Sethi ${ }^{1}$, J. Chipping ${ }^{1}$, S. Miles ${ }^{1}$, N. Shenker ${ }^{2}$,

S. Sami ${ }^{1}$, A. Macgregor ${ }^{1} .{ }^{1}$ University of East Anglia, Norwich Medical School,

Norwich, United Kingdom; ${ }^{2}$ Addenbrookes University Hospital, Rheumatology,

Cambridge, United Kingdom

Background: Chronic pain is debilitating and prevalent. Current non-pharmacological management of pain conditions such as Fibromyalgia Syndrome (FMS) are labour intensive to implement and poorly available, especially during the pandemic. There is an urgent need to develop widely adoptable, innovative treatment options for pain cohorts.

Virtual reality (VR) provides an innovative therapeutic tool, immersing users within a three-dimensional, interactive virtual environment with use of a headmounted display (HMD). Beneficial effects of VR have been demonstrated in acute pain ${ }^{1}$, with limited studies in chronic pain. Given the variation of available VR technologies, it is vital to investigate the impact of different VR characteristics on acceptability in specific chronic pain cohorts.

Objectives: This feasibility study aims to establish the acceptability of four different VR technologies in patients with FMS whilst undertaking a single interactive VR experience.

Methods: Patients with FMS were recruited through outpatient clinics at the Norfolk and Norwich University Hospital. Baseline questionnaires were used including the McGill pain questionnaire (MPQ-SF), pain visual analogue scale (VAS) and Revised Fibromyalgia Impact Questionnaire (FIQR). Subjective experience questionnaires collected acceptability data with 7-point Likert scale rating questions (strongly disagree to strongly agree). The simulation sickness questionnaire (SSQ) gained side-effect data (total severity score: $0-235$ ). Categorical data were described using frequencies; and continuous data using mean and standard deviation. Likert-scale data were dichotomised (rating $\leq 3$ : disagree, rating $\geq 5$ : agree).

Four VR systems representing the spectrum of commercially available technologies were used (seen in Figure 1). These possess different characteristics including screen resolution, processor speed, weight, strap and controller type. The VR experience used with each headset was co-developed alongside industry partners (Orbital Global). Participants are immersed within a naturalistic environment, situated on a wooden boat travelling slowly along a calm river surrounded by trees and hills. The interactive element involves the user shooting targets that appear using handheld controllers.

Results: 13 patients with FMS were included (mean age 41.8ะ15.6, 92.3\% female). Most had severe disease (mean FIQR 67.8 14.1 ) with moderate self-reported pain at baseline (mean MPQ $25.5 \pm 8.8$, VAS $6.0 \pm 1.7$ ). Most had no previous VR exposure (69.2\%). $100 \%$ of participants agreed that they would be open to using VR for future pain management (mean rating $6.5 \pm 0.7$ ) and that they would use VR regularly at home (mean rating 6.5 \pm 0.7 ). VR HMD comfort and enjoyment data are presented in Table 1. Mean ratings of comfort were high across the four HMDs (Gear VR: $4.9 \pm 1.7$, Oculus Go: $4.5 \pm 1.8$, Oculus Quest $5.3 \pm 1.9$, Oculus Rift $6.6 \pm 0.5$ ). Mean ratings of enjoyment with each HMD were also high (Gear VR: $5.4 \pm 1.6$, Oculus Go: $5.4 \pm 1.8$, Oculus Quest: $5.6 \pm 1.9$, Oculus Rift S: $6.6 \pm 0.5)$. Low levels of side effects were described with mean SSQ total scores ranging from 20.1 \pm 16.8 (Oculus Rift S) to $38.0 \pm 23.9$ (Gear VR).

Conclusion: Preliminary results indicate that FMS patients find VR acceptable, describing high ratings of comfort and enjoyment across the VR HMD spectrum. Side-effect frequency was low, with most settling after HMD removal. All participants were open to future use of VR for home-based pain management. REFERENCES:

[1] Dascal J, Reid M, Ishak WW, Spiegel B, Recacho J, Rosen B, Danovitch I. Virtual reality and medical inpatients: A systematic review of randomized, controlled trials. Innov Clin Neurosci 2017;14(1-2):14-21

Table 1. Subjective experience results across VR HMDs

\begin{tabular}{lcc}
\hline & Mean Likert scale ratings (\% agreement: rating $\geq 5)$ \\
\hline VR HMD & $\begin{array}{c}\text { Overall, I found the VR experience using this Overall, I enjoyed using this } \\
\text { VR headset }\end{array}$ \\
\hline Gear VR & $4.9 \pm 1.7(62 \%)$ & $5.4 \pm 1.6(77 \%)$ \\
Oculus Go & $4.5 \pm 1.8(54 \%)$ & $5.4 \pm 1.8(75 \%)$ \\
Oculus Quest & $5.3 \pm 1.9(77 \%)$ & $5.6 \pm 1.9(100 \%)$ \\
Oculus Rift S & $6.6 \pm 0.5(100 \%)$ & $6.6 \pm 0.5(100 \%)$ \\
\hline
\end{tabular}

